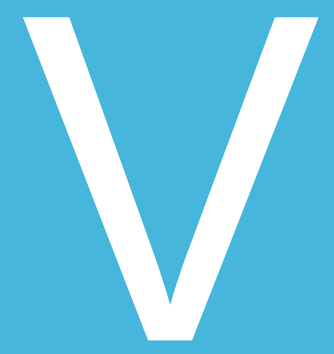

\title{
DEPRESIÓN Y CARACTERÍSTICAS
} DEMOGRÁFICAS ASOCIADOS EN ESTUDIANTES Y LÍDERES UNIVERSITARIOS DE LIMA METROPOLITANA

\section{Depression and demographic characteristics related to} university liders and students from Metropolitan Lima

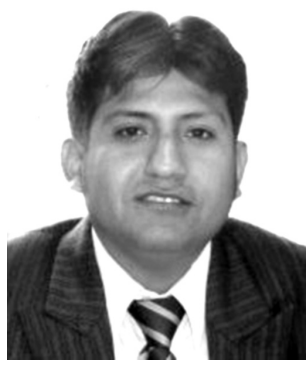

\section{Renzo Felipe Carranza Esteban}

Psicólogo por la Universidad Peruana Unión. Docente Auxiliar de la Facultad de Ciencias de la Salud por la Universidad Peruana Unión. Investigador y docente visitante. Actualmente se desempeña como director de investigación de la escuela académico profesional de Psicología en la sede Tarapoto de la Universidad Peruana Unión. 


\section{Resumen}

En años pasados la depresión era considerada como un signo, síntoma o síndrome; en la actualidad es una enfermedad como cualquier otra que ataca sin hacer distinciones de edad, sexo, estado civil, nivel socioeconómico. El objetivo del estudio fue determinar el nivel de depresión en los alumnos universitarios de Lima Metropolitana. Se tomó una muestra de 2005 sujetos de ambos sexos, entre 16 y 36 años. Se utilizó un diseño no experimental de tipo descriptivo. Instrumentos: Inventario de depresión de Beck Adaptado (BDI-IIA) por Carranza (2009). Los resultados muestran que existe depresión en los universitarios de Lima Metropolitana; 23.2\% presentan un nivel severo de depresión, asimismo un $24.1 \%$ un nivel moderado.

Palabras clave: Depresión estudiantil, enfermedad, síntomas.

\section{Abstract}

In past years depression was seen as a sign, symptom or syndrome, but today is a disease like any other that attacks without distinction of age, sex, marital status, socioeconomic status. The aim of the study was to determine the level of depression of University students in metropolitan Lima. A sample of 2005 students of both sexes was taking between 16 and 36 years, for which we used a non-experimental design a descriptive. Instruments: Beck Adapted (BDI- HA) by Carranza (2009). The results show that there is depression in Metropolitan Lima University students; $23.2 \%$ have a severe level of depression, and $24.1 \%$ also moderate.

Keywords: Student depression, disease, symptoms. 


\section{Introducción}

En el mundo son 121 millones de personas las que tienen actualmente depresión. Las investigaciones indican que, un diagnóstico y tratamiento temprano, podrían disminuir futuros episodios depresivos (OMS, 2001). En la actualidad es diez veces más frecuente que en el pasado, y aparece a una edad mucho más temprana. El promedio de edad del primer episodio depresivo de una persona en la década de los sesenta era de treinta años; mientras que hoy, se sitúa en los quince. (Suárez, 1998; véase en Pereyra y Mussi, 2005).

Según la Organización Mundial de la Salud (2001; véase en Gómez, Bohórquez, Pinto, Gil, Rondón y Díaz, 2004), la depresión, ocupa en la actualidad el cuarto lugar en la prevalencia de todas las enfermedades y, de acuerdo con las proyecciones para el año dos mil veinte, será la segunda enfermedad causante de muertes en el mundo y solo será superada por las enfermedades cardiovasculares. Se evidencia así que el índice de enfermedades ha ido incrementándose cada vez más y, a diferencia de años pasados donde la depresión era considerada como un signo, síntoma síndrome; en la actualidad es ya toda una enfermedad y ésta ha creciendo en forma drástica durante los últimos cuarenta años, en todos los países ricos y pobres del mundo.

Según el National Institute of Mental Healt (NIMH, 2001), cada año el $9.5 \%$ de la población estadounidense (aproximadamente 18.8 millones de adultos norteamericanos) padece de enfermedades depresivas. Desgraciadamente, muchas personas ignoran que la depresión es una enfermedad tratable.

Asimismo, el NIMH (2001), menciona que el trastorno depresivo es una enfermedad, que afecta el organismo (cerebro), el ánimo, la manera de actuar, la forma en que una persona come y duerme. Afecta cómo uno se valora a sí mismo (autoestima) y la forma en que uno piensa. Asimismo un trastorno depresivo no es lo mismo que un estado pasajero de tristeza y no indica debilidad personal, como tampoco es una condición de la cual uno puede liberarse voluntariamente.

Por otro lado, un estudio realizado en Estados Unidos reportó que un alto porcentaje de los 19 millones de adultos que sufren depresión son estudiantes universitarios. Dentro de esta franja, el 30\% de los estudiantes universitarios son de primer año, ellos informan sentirse abrumados por la vida universitaria y el $40 \%$ refiere que ha buscado ayuda de su centro de orientación estudiantil. En tal sentido, si no se trata a tiempo la depresión, puede causar trastornos alimenticios, abuso de alcohol y drogas e incluso suicidio (National Institute of Mental Healt, 2001). 
El reporte mundial de la salud (OMS 2001), indicó el incremento de desórdenes de salud mental, como la violencia, depresión, ansiedad, suicidio, esquizofrenia y el abuso de drogas; reconociendo dramáticamente a la depresión como el que tiene mayor prevalencia, afectando el 5,8\% de los varones del mundo y a 9,5\% de las mujeres (Gómez, Bohórquez, Pinto, Gil, Rondón y Díaz, 2004).

\section{La depresión}

En los últimos años la depresión ha sido catalogada como uno de los problemas que más sufrimiento causa en las personas y en distintos grados afecta a un porcentaje muy alto de la población sin diferencia de edad, género y nivel socioeconómico; convirtiéndola en uno de los principales motivos de consulta clínica.

Manelic y Ortega (1995), mencionan que en los estudiantes universitarios la depresión puede constituir un problema de real importancia, pues al malestar psicológico del individuo se agrega la imposibilidad de obtener un rendimiento académico satisfactorio debido a que las funciones cognoscitivas se alteran sensiblemente cuando se presenta esta entidad nosológica (depresión). Además, el estudiante universitario se enfrenta con una nueva realidad que, muchas veces, lo abruma y parece superar sus posibilidades de adaptación. (Riveros, Hernández y Rivera, 2007).

La depresión puede ser desencadenada por cualquier cambio de vida estresante, incluso si es un cambio drástico en la vida normal y habitual, por ejemplo, el cambio es elegido como el caso de una carrera universitaria. El ingreso a la universidad suele ser el primer cambio importante en la vida de un joven adulto. En esta fase de transición, los estudiantes suelen enfrentar problemas tales como: nuevos arreglos de vida, necesidad de supervisar el horario de uno mismo, atender las necesidades propias (horarios para comer, lavar la ropa, dormir y trabajar), presión académica, responsabilidades financieras, cambios en las relaciones con la familia y los amigos, presiones debido a las relaciones nuevas, aumento de la consciencia de identidad sexual, preocupaciones sobre la vida después de la graduación, etc.

De otro lado, es importante diferenciar la depresión de la tristeza. La tristeza es un estado de ánimo pasajero, provocado por una situación concreta, como por ejemplo, la muerte de un ser querido. Cuando este estado de ánimo perdura en el tiempo, podemos estar frente a una depresión. Según el NIMH (2001), los siguientes síntomas pueden considerarse como indicadores de la depresión: un estado de ánimo triste o ansioso persistente al igual que sensaciones de desesperanza, el pesimismo, la culpabilidad, la impotencia, la pérdida de interés en actividades que resultaban placenteras, el cansancio 
-fatiga-, disminución de energía, problemas de concentración y memoria, dificultad para tomar decisiones, autocrítica, el despertarse en la madrugada o dormir demasiado, cambios en el apetito, pensamientos de muerte o intentos de suicidio, inquietud, irritabilidad, dolores de cabeza y alteraciones digestivas que no responden a tratamiento médico; son síntomas que se pueden considerar como indicadores de la depresión.

El paso por la universidad debería ser una experiencia satisfactoria; sin embargo, en muchos casos no suele ser así, pues cada vez más se exige al alumno en el ambiente universitario, no solo desde las aulas, sino también la sociedad genera en el alumno cierto desequilibrio emocional; es así que en estudios realizados en países como Japón reportan que un $20 \%$ universitarios padecen depresión (Vázquez, 2005).

\section{Definición de depresión}

Respecto a la depresión, Beck (1976), menciona que debe a un desorden del pensamiento, en la que el depresivo distorsiona la realidad viéndose de forma negativa en la triada cognitiva: el mismo, el mundo y el futuro; además supone la activación de signos y síntomas como consecuencia de la activación de patrones cognitivos negativos.

Robert y Lamontegne (1977), consideran que la depresión es un estado de tristeza, acompañada de una marcada disminución del sentimiento de valor personal de una dolorosa consecuencia de disminución de actividad mental psicomotriz y orgánica, por otro lado, Fremman y Oster, citado por Salmuri y Skoknic (2005), postulan que la depresión es un trastorno conocido desde hace tiempo, mencionando que es el problema psicológico de nuestros días, considerado como el resfriado de los trastornos emocionales. Cottraux citado por Vázquez y Sanz (1999), menciona que la depresión es una síndrome caracterizado por un estado de ánimo que se traduce en la expresión verbal y no verbal de sentimientos tristes, acompañados de ansiedad e irritabilidad, dichas características pueden alterar múltiples funciones como el sueño y el apetito, la energía y el impulso sexual, la motivación se traduce en un déficit del interés y de la capacidad para experimentar placer, el deseo de evadirse mediante el suicido es frecuente, la evitación de los contactos sociales y la dependencia respecto a los demás lleva recientemente a un rechazo por el miedo. Los comportamientos activos y productivos se encuentran disminuidos y los pensamientos negativos se encuentran incrementados.

Vázquez citado por Vázquez y Sanz (1991), menciona sobre el conjunto de síntomas -indicadores- que componen el síndrome depresivo, se pueden reducir a cuatro grandes núcleos: 
Síntomas anímicos. Los síntomas -indicadores- que comprenden este núcleo son la disforia, sentimientos de abatimiento, pesadumbre, llanto, infelicidad, e incluso irritabilidad y tristeza.

$\checkmark$ Síntomas motivacionales. Los síntomas -indicadores- que comprenden este núcleo son la apatía, la indiferencia, la anhedonia, disminución en la capacidad de disfrute- desalineo personal, ganas de abandonar el estudio y falta de toma de decisiones junto con el estado de ánimo deprimido, el síntoma principal de un estado depresivo, el levantarse de la cama por las mañanas o asearse pueden convertirse en tareas casi imposibles y, en muchos casos, se abandonan los estudios o el trabajo. Asimismo, tomar decisiones cotidianamente como decidir la ropa para vestirse puede convertirse en una tarea casi insalvable.

$\checkmark$ Síntomas cognitivos. Los síntomas -indicadores- que comprenden este grupo son la autodepreciación, la autoculpación, y la pérdida de autoestima.

$\checkmark$ Síntomas físicos. Los síntomas que comprenden este núcleo son la perdida de sueño, la fatiga, la pérdida de apetito, náuseas, vómitos, estreñimiento, micción dolorosa, visión borrosa evidencia de disminución de la actividad y el deseo sexual.

$\checkmark$ Síntomas interpersonales. El síntoma -indicador-principal de este núcleo es que la persona deprimida no tiene por relacionarse con los demás.

\section{Materiales y Métodos}

\section{Diseño y Tipo de Investigación}

La investigación que se llevó a cabo se define como descriptiva; utilizando un diseño no-experimental de carácter transversal, dado que la recolección de datos es en un momento único (Hernández, Fernández y Baptista, 2004; Sampieri, Collado y Baptista, 1997). Asimismo, se trata de un diseño ex-post-facto, en el que no existe manipulación de las variables, sino que al momento de la investigación, la variable depresión se encuentra presente en algún grado de desarrollo y cuya variación no depende del investigador.

\section{Participantes}

Estuvo conformada por estudiantes universitarios, tanto de universidades privadas como nacionales, de Lima Metropolitana. El número de personas que se tomó en cuenta, es de 2005 estudiantes universitarios de ambos sexos cuyas edades ocilaban entre 16 y 36 años. 


\section{Instrumentos}

Inventario de Depresión de Beck Adaptado (BDI-IIA) conformada por 21 ítems (Carranza, 2009).

\section{Resultados}

Propiedades psicométricas del Inventario de Depresión de Beck Adaptado (BDI-IIA)

\section{Confiabilidad}

En cuanto a determinar la confiabilidad en su modalidad de consistencia interna se estimó el coeficiente Alfa de Cronbach, obteniéndose como resultado para la escala total un valor de 0.878 para el Inventario de Depresión de Beck Adaptado (Tabla 1), por lo que se puede concluir que el instrumento permite obtener puntajes confiables.

Tabla 1

Estimaciones de confiabilidad mediante el coeficiente Alfa de Cronbach para el Inventario de Depresión de Beck Adaptado (BDI-IIA)

\begin{tabular}{ccc}
\hline $\begin{array}{c}\mathrm{N}^{\circ} \text { de } \\
\text { items }\end{array}$ & $\begin{array}{c}\text { Alfa de } \\
\text { Cronbach }\end{array}$ & $\begin{array}{c}\mathrm{N}^{\circ} \text { de } \\
\text { casos }\end{array}$ \\
\hline 21 & 0.878 & 2005 \\
\hline
\end{tabular}

\section{Validez}

Como se puede observar en la Tabla 2, la Prueba KMO y Prueba de Barlett, en conclusión, valores bajos del índice KMO desaconsejan la utilización de Análisis Factorial. En la Tabla 2 se observa que el índice KMO es de 0.929 siendo un valor meritorio para obtener el análisis factorial lo que indica que el instrumento presenta validez. 


\section{Tabla 2}

Prueba KMO y prueba de Barlett

\begin{tabular}{lll}
\hline $\begin{array}{l}\text { Medida de adecua- } \\
\text { ción muestral de Kai- }\end{array}$ & 0.929 \\
ser-Meyer-Olkin. & & \\
\hline $\begin{array}{l}\text { Prueba de esferici- } \\
\text { dad de Bartlett }\end{array}$ & $\begin{array}{l}\text { Chi-cuadrado } \\
\text { aproximado }\end{array}$ & 10844.609 \\
& & \\
& gl & 210 \\
& Sig. & 0.00 \\
\hline
\end{tabular}

\section{Análisis descriptivo de las variables de estudio}

Se percibe en la Tabla 3 que un 53\% de la población de estudiantes universitarios de Lima Metropolitana pertenece al género femenino; de otro lado se aprecia que un $77.5 \%$ de estudiantes universitarios de Lima Metropolitana que se ubican entre las edades de 16 y 22 años de edad presentan mayores niveles de depresión.

Tabla 3

Niveles de depresión por características demográficas (porcentaje entre paréntesis)

\begin{tabular}{|c|c|c|c|c|c|c|c|c|c|c|}
\hline \multirow{2}{*}{ Características } & \multicolumn{10}{|c|}{ Nivel de depresión } \\
\hline & \multicolumn{2}{|c|}{ Mínima } & \multicolumn{2}{|r|}{ Leve } & \multicolumn{2}{|c|}{ Moderada } & \multicolumn{2}{|c|}{ Severa } & \multicolumn{2}{|c|}{ Total } \\
\hline Masculino & 264 & (13.2) & 247 & (12.3) & 220 & (11.0) & 205 & (10.2) & 936 & (46.7) \\
\hline Femenino & 262 & (13.1) & 283 & (14.1) & 264 & (13.2) & 260 & (13.0) & 1069 & (53.3) \\
\hline \multicolumn{11}{|l|}{ Edad } \\
\hline $16-22$ & 388 & (19.4) & 420 & (20.9) & 388 & (19.4) & 357 & $(17.8)$ & 1553 & (77.5) \\
\hline $23-29$ & 118 & (5.9) & 100 & (5.0) & 89 & (4.4) & 99 & (4.9) & 406 & (20.2) \\
\hline $30-36$ & 20 & (.9) & 10 & (.4) & 7 & (.3) & 9 & (.4) & 46 & (2.3) \\
\hline Total & 526 & (26) & 530 & (26) & 484 & (24) & 465 & (23) & 2005 & (100) \\
\hline
\end{tabular}

Por último, queremos Ilamar la atención sobre los resultados obtenidos; en la Tabla 04 se observa que los estudiantes de la Universidad Peruana Unión, Universidad TELESUP, Universidad de Lima, Universidad Cayetano Heredia, Universidad Nacional de Ingeniería y la Universidad San Juan Bautista presentan un nivel mínimo de depresión y, a su vez, las universidades que 
tienen mayores niveles de depresión son: Universidad Nacional Mayor de San Marcos, Universidad Cayetano Heredia, Universidad Tecnológica del Perú, Universidad San Ignacio de Loyola y la Universidad Nacional del Callao, lo que indica que se va cumpliendo las predicciones de la Organización Mundial de la Salud.

Es posible que esto se deba a las exigencias académicas a que son sometidos los estudiantes universitarios; así, investigaciones realizadas reportan que las facultades de ingeniería y de ciencias de la salud son las que están mayormente predispuestas a decaer emocionalmente en algún momento de la vida siendo la tensión y factor predisponente (Espinosa y Roman, 1993; Stephanou, 2011).

Tabla 4

Niveles de depresión por universidades (porcentaje entre paréntesis)

\begin{tabular}{|c|c|c|c|c|c|c|c|c|c|c|}
\hline \multirow{3}{*}{$\begin{array}{c}\text { Universidades } \\
\text { Universidad Peruana Unión }\end{array}$} & \multicolumn{10}{|c|}{ Nivel de depresión } \\
\hline & \multicolumn{2}{|c|}{ Mínima } & \multicolumn{2}{|c|}{ Leve } & \multicolumn{2}{|c|}{ Moderada } & \multicolumn{2}{|c|}{ Severa } & \multicolumn{2}{|c|}{ Total } \\
\hline & 164 & (33.5) & 146 & (29.9) & 93 & (19.0) & 86 & (17.6) & 489 & $(100.0)$ \\
\hline Universidad Federico Villarreal & 34 & (20.4) & 44 & (26.3) & 45 & (26.9) & 44 & & 167 & \\
\hline $\begin{array}{l}\text { Universidad Enrique Guzmán y Valle } \\
\text { - La cantuta }\end{array}$ & 26 & (25.7) & 32 & (31.7) & 20 & $(19.8)$ & 23 & $(22.8)$ & 101 & $(100.0)$ \\
\hline Universidad Privada TELESUP & 20 & (31.7) & 13 & $(20.6)$ & 18 & (28.6) & 12 & (19.0) & 63 & $(100.0)$ \\
\hline Universidad Mayor de San & 28 & (21. & 28 & $(21$. & 29 & .0) & 47 & & 132 & \\
\hline Universidad de Lima & 35 & (39.3) & 31 & $(34$. & 13 & (14. & 10 & & 89 & \\
\hline $\begin{array}{l}\text { Universidad Pontificia Católica del } \\
\text { Perú }\end{array}$ & 12 & (21.4) & 17 & (30.4) & 14 & $(25.0)$ & 13 & $(23.2)$ & 56 & $(100.0)$ \\
\hline Universidad Cayetano Heredia & 23 & $(40.4)$ & 7 & $(12.3)$ & 9 & & 18 & & 57 & \\
\hline Univers & 16 & $(28$. & 19 & $(33.3)$ & 12 & .1) & 10 & $(17.5)$ & 57 & \\
\hline Universidad Ricard & 13 & $(13.0)$ & 38 & $(38.0)$ & 31 & (31.0) & 18 & $(18.0)$ & 100 & \\
\hline Universidad Tecnológica del Perú & 8 & $(9.2)$ & 20 & $(23.0)$ & 28 & $(32.2)$ & 31 & $(35.6)$ & 87 & $0.0)$ \\
\hline Universidad Inca Gar & 21 & $(20.4)$ & 25 & $(24.3)$ & 36 & $(35.0)$ & 21 & (20.4) & 103 & $(100.0)$ \\
\hline Universidad Privada Norbeth Wiener & 12 & $(16.4)$ & 19 & $(26.0)$ & 23 & (31.5) & 19 & $(26.0)$ & 73 & $(100.0)$ \\
\hline Universidad Nacional de Ingeniería & 37 & $(37.0)$ & 20 & $(20.0)$ & 18 & $(18.0)$ & 25 & $(25.0)$ & 100 & $(100.0)$ \\
\hline $\begin{array}{l}\text { Universidad Nacional Agraria La } \\
\text { Molina }\end{array}$ & 11 & $(17.7)$ & 22 & $(35.5)$ & 13 & (21.0) & 16 & $(25.8)$ & 62 & $(100.0)$ \\
\hline Universidad San Ignacio de Loyola & 11 & $(23$. & 7 & $(15$. & 13 & $(28.3)$ & 15 & (32. & 46 & \\
\hline Universidad Alas Peruanas & 11 & $(23.4)$ & 11 & $(23.4)$ & 17 & $(36.2)$ & 8 & $(17.0)$ & 47 & $(100.0)$ \\
\hline $\begin{array}{l}\text { Universidad Femenina del Sagrado } \\
\text { Corazón (Unife) }\end{array}$ & 12 & (23.1) & 6 & (11.5) & 22 & $(42.3)$ & 12 & $(23.1)$ & 52 & $(100.0)$ \\
\hline Universidad San Juan Bautista & 16 & (31. & 7 & $(13$. & 16 & & 12 & (23.5) & 51 & $(100.0)$ \\
\hline Universidad Nacional del Callao & 10 & & 7 & $(14.6$ & 8 & $(16.7)$ & 23 & (47.9) & 48 & $(100.0)$ \\
\hline Universidad San Martín de Porres & 6 & $(24.0)$ & 11 & 44.0 & 6 & $(24.0)$ & 2 & $(8.0)$ & 25 & $(100.0)$ \\
\hline
\end{tabular}




\section{Discusión}

La importancia de este trabajo radica en la evidencia empírica que aporta la salud mental, demostrando que en la actualidad existe depresión en los estudiantes universitarios de Lima Metropolitana. Se percibe, entonces, a la depresión como una enfermedad que ataca como cualquier enfermedad sin hacer distinciones de edad, sexo, estado civil o nivel socioeconómico. Confirmamos que la depresión se está convirtiendo en una enfermedad mortal la misma que fue corroborada por la Organización Mundial de la Salud (2001; Gómez, Bohórquez, Pinto, Gil, Rondón y Díaz, 2004). La depresión, ocupa en la actualidad el cuarto lugar en la prevalencia de todas las enfermedades y, de acuerdo con las proyecciones para el año 2020, será la segunda enfermedad causante de muertes en el mundo y solo será superada por las enfermedades cardiovasculares.

Asimismo, la depresión en universitarios se está convirtiendo en un fenómeno frecuente, dado que se deteriora significativamente la adaptación psicosocial en diferentes contextos geográficos, y económico-sociales en los cuales existen universitarios.

Resultados similares se muestran en la investigación desarrollada por Arrivillaga, Cortes, Goicochea y Lozano (2003), quienes realizaron un estudio de carácter no experimental, descriptivo y transeccional, donde el objetivo fue describir las características de la depresión en jóvenes universitarios. La población de estudio estuvo conformada por 4421 jóvenes, hombres y mujeres estudiantes de carreras de pregrado de la Pontificia Universidad Javeriana de la ciudad de Cali, tomando una muestra de 221 estudiantes universitarios y se tuvo como resultado, que el $43 \%$ de las mujeres y el $18 \%$ de los hombres presentaban algún tipo de depresión. Posteriormente, se amplió la investigación donde la muestra estuvo constituida por 245 jóvenes (92 hombres y 153 mujeres), obteniendo como resultados la presencia significativa de depresión en un $30 \%$, según el Inventario de Depresión de Beck (BDI) y $25 \%$, según la Escala Autoaplicada de Zung (EAZ).

Finalmente, tomando en cuenta los resultados obtenidos, esperamos el diseño e implementación de programas de prevención e intervención de la depresión.

\section{Conclusiones}

De acuerdo a los datos y resultados presentados en esta investigación, se arriba a las siguientes conclusiones: 


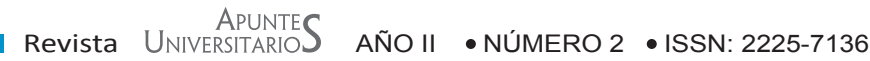

1. Respecto a nuestro objetivo general, se encuentra que existe depresión en los alumnos y líderes universitarios de Lima Metropolitana.

2. En relación a nuestro primer objetivo específico, se encontró que los jóvenes líderes universitarios que se encuentran entre 16-22 años presentan depresión severa, a diferencia de los que se encuentran situados entre 30-36 años respectivamente.

3. Referente a nuestro segundo objetivo, se encontró que los estudiantes universitarios de Lima Metropolitana que presentan mayores niveles de depresión pertenecen al género femenino.

Renzo Felipe Carranza Esteban

Universidad Peruana Unión email:renzoc@upeu.edu.pe

Recibido: 06 de Julio de 2012 Aceptado: 18 de Octubre de 2012 


\section{Renzo Felipe Carranza Esteban}

\section{Referencias}

Beck, A., Steer, R., GK, Brown. (2006). Inventario de Depresión de Beck. Argentina: Paidos.

Carranza, R. (2009). Propiedades psicométricas del inventario de depresión de Beck (BDI-II) para universitarios de Lima Metropolitana. Tesis para optar el grado de Psicólogo. Lima, Perú: Universidad Peruana Unión.

Espinosa, J. y Roman, T. (1993). Actitudes hacia la Ciencia en estudiantes universitarios de Ciencias. Enseñanza de las Ciencias, 1993, 11(3), 297-300.

Gómez, C., Bohórquez, A., Pinto, D., Gil, J., Rondón, M., y Díaz, N. (2004). Prevalencia de depresión y factores asociados con ella en la población colombiana. Revista Panamericana de Salud Pública, 16(6), 378-386.

Hernández, R., Fernández, C., y Baptista, P. (1997). Metodología de la investigación. México: Mc Graw-Hill.

Manelic, H. y Ortega, H. (1995). La depresión en estudiantes universitarios de la Escuela Nacional de Estudios Profesionales Plantel Aragón. Revista de Salud Mental, 18(2), 31-34.

National Institute of Mental Health. (2002). Depresión. Recuperado de http://www.nimh.nih. gov/publicat/spDep3561.cfm

Pereyra, M. y Mussi, C. (2005). Sea Feliz, ¿cómo vencer la depresión y controlar la ansiedad? Montemorelos, Nuevo León: Ed. Montemorelos México.

Robert, S. y Lamontagne, Y. (1977). Depresión. Principios y aplicaciones de las terapias de la conducta. Madrid: Debate.

Salmuri, F. y Skoknic, V. (2005). Efectos conductuales de la educación emocional en alumnos de educación básica. Revista de Psicología Universidad de Chile, 14(001), 9-28.

Stephanou, G. (2001). Students classroom emotions: Socio-cognitive antecedents and school performance. Journal of Research in Educational Psychology, 9(1), 5-48.

Riveros, M., Hernández, H., y Rivera, J. (2007). Niveles de depresión y ansiedad en estudiantes universitarios de Lima Metropolitana. Revista de Investigación en Psicología, 10(1).

Vázquez, C. y Sanz, J. (1991). Trastornos depresivos: Trastornos del estado de ánimo: Teorías psicológicas. En Belloch A, Sandin, B y Ramos, F. Manual de Psicopatología. (vol. I-II, pp. 342-378). España: McGraw-Hill.

Vázquez, C. y Sanz, J. (1999). Fiabilidad y validez de la versión española del Inventario para la Depresión de Beck de 1978 en pacientes con trastornos psicológicos. Revista especializada en la psicología clínica y de la salud: trastornos psicológicos, saludenfermedad, prevención y atención a los diferentes niveles asistenciales (primaria, secundaria y terciaria), 1(10), 59-81. 